\title{
Politique
}

\section{Les scandales politiques aux États-Unis : carences d'un président ou d'un système politique}

\section{Edmond Orban}

Numéro 12, automne 1987

Mouvements et acteurs

URI : https://id.erudit.org/iderudit/040569ar

DOI : https://doi.org/10.7202/040569ar

Aller au sommaire du numéro

Éditeur(s)

Société québécoise de science politique

ISSN

0711-608X (imprimé)

1918-6584 (numérique)

Découvrir la revue

Citer cet article

Orban, E. (1987). Les scandales politiques aux États-Unis : carences d'un président ou d'un système politique. Politique, (12), 93-110.

https://doi.org/10.7202/040569ar d'utilisation que vous pouvez consulter en ligne.

https://apropos.erudit.org/fr/usagers/politique-dutilisation/ 


\title{
LES SCANDALES POLITIQUES AUX ÉTATS-UNIS: CARENCES D'UN PRÉSIDENT OU D'UN SYSTÈME POLITIQUE
}

\author{
Edmond Orban \\ Université de Montréal
}

Pour la majorité du public américain et même des observateurs les plus avertis de la présidence aux États-Unis, l'affaire dite Irangate est une surprise d'autant plus grande que les sondages de l'opinion publique s'avéraient exceptionnellement favorables pour le Président.

Et pourtant, si l'on y regarde de plus près, ce scandale n'est peut-être que la conséquence logique d'un phénomène aux dimensions multiples. Parmi celles-ci on retrouve plusieurs variables: la personnalité du Président et de ses conseillers et la partie la plus visible du système politique à savoir ce qu'il est convenu d'appeler le «presidential advisory system ${ }^{1}$ ou ensemble d'institutions et personnes ayant pour fonction principale de conseiller le chef d'État.

Notons, qu'en général, en cas de réussite nationale spectaculaire ou de crise majeure, l'on a tendance à conférer beaucoup

1. Voir Thomas Cronin et Sanford Greenberg, The Presidential Advisory System. New York, Harper and Row, 1969.

Politique, 12 (Automne 1987). 
trop d'importance à la variable personnalité du président. Certes, son caractère d'unique élu (avec le vice-président) face au Congrès y contribue grandement. Mais c'est aussi le résultat d'un processus d'hypersimplification des données, que les mass média ont contribué à maintenir, sinon à accentuer. Jamais l'image n'a joué un tel rôle et exercé une telle influence sur un public à la fois passif et réceptif. Certaines émissions de radio et télévision et plusieurs grands quotidiens et hebdomadaires américains, parmi les mieux informés et les plus critiques ont, en partie seulement, contrebalancé le processus en question. Ce sont d'ailleurs les mêmes que l'on retrouve lors du Watergate. Quoiqu'il en soit, une conséquence de cette situation, c'est que le facteur charismatique, dans un cas comme celui du Président actuel surtout, tend à rejeter dans l'ombre d'autres éléments essentiels de la politique, relevant davantage de l'infrastructure qui sert de support au titulaire de la fonction présidentielle.

\section{Présidence et limites de l'infrastructure politique.}

Il faudrait donc concentrer notre attention prioritairement sur certaines composantes de cette infrastructure. Et nous formulons un premier constat, évident en soi mais lourd de conséquences pour l'organisation du pouvoir: la fonction présidentielle d'une grande puissance telle que les États-Unis s'avère de plus en plus écrasante pour son unique responsable politique ${ }^{2}$. De nombreux observateurs sont d'accord pour dire que les problèmes de politique intérieure et extérieure ont pris des proportions d'une telle ampleur et d'une telle complexité qu'aucun système ne peut plus prétendre

2. C'est ce que soulignait déjà en 1968 Louis Koenig dans son ouvrage The Chief Executive où il analysait systématiquement l'ensemble des responsabilités présidentielles (New York, Harcourt). Voir, plus récemment, Richard Neustadt, Presidential Power, New York, Wiley, 1980 et Thomas Gronin, Rethinking the Presidency, Boston, Little Brown, 1982. 
les résoudre avec un succès assuré, malgré l'importance des ressources humaines et matérielles dont dispose l'exécutif de ce pays.

À première vue pourtant les charges de l'État fédéral auraient dû diminuer car rarement depuis Hoover (avant la grande crise) un président n'a autant parlé de désengagement de l'État dans les affaires sociales et économiques, de déréglementation, de privatisation et de décentralisation. En réalité, quel que soit le parti au pouvoir et les solutions apportées, les exigences d'une société industrialisée de cette importance provoquent d'autant plus de tensions à l'intérieur du système que les output sont plus limités. Il y a un décalage croissant entre l'augmentation des dites exigences et l'accessibilité aux ressources de nature à les satisfaire. Ce phénomène s'observe particulièrement dans la fonction budgétaire où les dépenses dépassent le mille milliards de dollars alors que les recettes ne suivent pas.

Confrontés avec les problèmes internes, les États-Unis éprouvent en plus, et malgré la liquidation de la guerre au Vietnam, beaucoup de difficultés à traiter les problèmes de politique extérieure spécialement dans le Proche-Orient et en Amérique centrale. Or, l'institution présidentielle se trouve au centre même du système décisionnel. C'est elle qui, en premier lieu et presque exclusivement, subit le choc en retour des décisions prises, en particulier de leur échec, quelle que soit l'importance de ces dernières par rapport à l'ensemble. La concentration de l'impact sur la présidence semble, dans ce système, épargner le Congrès (élu lui aussi). La responsabilité de ce dernier se trouve en quelque sorte diluée dans deux assemblées marquées par la fragmentation des intérêts, le sectionalisme régional et souvent un manque de cohésion politique (pas seulement au sens partisan du terme).

Dans un tel contexte, contrairement à ce que l'on peut observer en régime parlementaire, le chef de l'exécutif ne dispose pas d'un appui comparable au sein du législatif. D’où la nécessité 
de créer des institutions nouvelles et spéciales pour combler en partie cette lacune, tout en échappant au contrôle trop souvent jugé négatif du dit législatif. On voit ici le danger que ce phénomène présente et comment il peut ouvrir facilement la porte à l'illégalité au nom de l'efficacité, surtout en politique étrangère où, de toute façon, le Congrès a trop souvent renoncé à exercer en profondeur et systématiquement une fonction de contrôle digne de ce nom ${ }^{3}$. Mais, en définitive, le Congrès est-il ou peut-il être équipé pour remplir cette tâche essentielle? Question fondamentale que l'on ne peut esquiver lorsque l'on aborde le scandale Irangate. Question qui a aussi le mérite de situer le débat dans un cadre plus large et d'examiner les responsabilités politiques d'une façon moins unilatérale ou trop centrée sur une ou quelques variables seulement, alors que les carences présidentielles sont dans une large mesure également le résultat, au moins indirect, de carences du Congrès et des partis politiques. Tout cela rend évidemment la tâche de l'observateur infiniment plus compliquée car son cadre spatiotemporel comporte un nombre accru de variables d'ordre systémique.

Second constat évident découlant de ce qui précèdè: étant donné la quantité, la variété et la complexité des décisions à prendre, dont certaines dans un délai souvent très court, le président ne peut fonctionner sans une division des tâches très poussée, sans un ensemble de conseillers de grande valeur pour contrôler au moins les rouages clefs de son organisation. On dit souvent aux États-Unis qu'elle est à tout jamais révolue l'époque où l'on pouvait fonctionner avec des Présidents relativement faibles et mal conseillés,

3. Et ceci, en dépit des réformes opérées au Congrès, notamment avec le "Legislative Reorganization Act " de 1970 où le Congrès essaye de changer ses procédures et son organisation pour mieux exercer ses responsabilités envers l'exécutif. D'autres réformes ont suivi, spécialement après la guerre du Vietnam et l'affaire Watergate mais avec des résultats mitigés. 
comme ceux que l'on a connus, par exemple, entre la fin de la première guerre mondiale et la grande crise ${ }^{4}$.

Limitons-nous pour l'instant au problème des institutions destinées à conseiller le Président (Presidential Advisory System). Elles ont fait l'objet d'innombrables études et commissions d'enquête, tant gouvernementales que privées (surtout universitaires). Celles-ci ont contribué à plusieurs améliorations techniques et réduit des lacunes d'ordre administratif. Mais elles n'ont jamais véritablement résolu le problème crucial de la coordination ${ }^{5}$, et du contrôle par conséquent, des activités de ces énormes machines administratives que constituent les départements, agences et commissions dont les "grandes commissions indépendantes» à pouvoir réglementaire où les membres une fois nommés ne font l'objet que d'un contrôle indirect de l'exécutif. Autre cas spécial: la C.I.A., théoriquement contrôlée par le Congrès ${ }^{6}$ et dont les activités sont censées être coordonnées au sein du C.N.S. Cette agence pose des problèmes insolubles qui ressortent à chaque crise importante dont celle de l'Irangate.

4. Voir notamment James Barber, The Presidential Character, New Jersey, Prentice Hall, 1972. Dans sa typologie des personnalités présidentielles, il souligne qu'il n'y aura plus de place dans ce système pour des présidents passifs même du type positif (ce qui s'appliquerait au Président Reagan). Pour un point de vue analogue, voir, avant l'affaire Watergate, Erwin Hargrove, Presidential Leadership, Personnality and Political Style, NewYork, Macmillan, 1968.

5. Sous Truman, en 1951, on a créé au C.N.S., un «Psychological Strategy Board", comportant le directeur de la C.I.A. et les chefs de département de la défense et des affaires étrangères.

6. En vertu notamment des amendements apportés au Foreign Assistance Act de 1974, la C.I.A. est théoriquement contrôlée par plusieurs commissions à la Chambre des Représentants et au Sénat, en particulier par les deux "Commission des Armées" et les deux "Commissions des Affectations budgétaires" (Appropriations Committees). Depuis 1976 pour le Sénat et 1977 pour la Chambre des Représentants, il existe des «Select Committees» (commissions nommées dans un but spécifique) ayant dans leurs attributions les activités de renseignement. 
Quant au problème général de la division des tâches et de la responsabilité de la coordination, les partisans du régime parlementaire soulignent les avantages dont dispose un premier ministre pouvant s'appuyer sur une équipe de ministres élus comme lui, responsable devant le Parlement, servant d'intermédiaire pour les électeurs et les groupes d'intérêt. Dans un tel cadre, il est moins difficile de procéder à une certaine division des tâches politiques et administratives, tout en gardant un minimum de cohésion à l'intérieur d'un cabinet élargi. Aux États-Unis, le cabinet n'existe que nominalement, il n'a ni la légitimité ni les responsabilités majeures de l'organisme précité, les chefs de département du cabinet présidentiel n'étant pas élus. Le Président ne s'appuie donc pas sur une équipe servant d'articulation entre lui et le législatif, entre lui et la population. Dans ce système, par contre, on met davantage l'accent sur des «institutions de conseil» plus élaborées, plus spécialisées, composées surtout (au sommet) de technocrates venant majoritairement des entreprises privées. Ainsi, idéalement l'on peut aller chercher les «meilleurs cerveaux» du pays alors que le premier ministre parlementaire doit choisir ses principaux conseillers dans un bassin beaucoup plus limité et d'une autre nature.

Or, que révèlent les analyses effectuées au sujet de la formation et de l'expérience des principaux conseillers présidentiels? Elles dégagent un profil type, celui d'un conseiller venant surtout des grandes entreprises industrielles ou du monde des affaires ${ }^{7}$ et en partie des grandes universités. À cet égard il y a une espèce d'osmose: professeur d'université - cadres supérieurs dans les entreprises - conseillers présidentiels, la même personne étant prise dans cette «mobilité triangulaire». Shultz est un exemple

7. Ceci est vrai, même sous Kennedy qui avait la réputation d'ouvrir davantage son éventail de conseillers, voir H. Rowen, The Free Enterprises: Kennedy. Johnson and the Business Establishment, New York, Putnam's Sons, 1964. 
de ce type de conseiller, le plus expérimenté mais pas forcément le plus écouté.

Cette méthode de sélection des principaux conseillers résulte donc des limites imposées par la constitution d'un régime présidentiel et en même temps d'une culture politique où l'on est soucieux avant tout d'efficacité (souvent à court terme). On croit que les méthodes utilisées dans l'entreprise privée sont les plus rentables, parce que fondées sur une technologie éprouvée et un mode de gestion diamétralement opposé au modèle bureaucratique public jugé défavorablement.

Quelles que soient ses qualités de gestionnaire, ce type de conseiller n'est pas représentatif des intérêts de l'ensemble des régions et des classes sociales. Certes, le problème du caractère représentatif ou non des conseillers ne nous concerne pas directement ici. Mais l'on doit cependant constater que les carences à ce sujet les rendent souvent incapables d'exprimer adéquatement (et sensiblement) les besoins et les opinions de très larges segments de population. Nous avons un système par conséquent coupé de sa base et tôt ou tard ceci doit avoir un impact sur l'efficacité et le fonctionnement du système politique et en particulier de sa partie la plus visible. Mais celle-ci n'est, on le voit que la pointe de l'iceberg.

Cet ensemble de conseillers se divisent un certain nombre de tâches et se répartissent dans des organes de conseil à vocation diverse nettement spécialisés ou jouissant d'attribution transcendant la politique intérieure et extérieure. Les conseillers les plus mobiles et souvent les plus polyvalents proviennent du Bureau de la Maison Blanche. Ainsi sous la présidence Reagan, Donald Regan et avant lui James Baker, considérés comme les chefs d'état-major du président, fonctionnaient dans le cadre de cette institution. Ajoutons à cela le Conseil national de Sécurité, le Conseil économique, le Bureau du Budget et de l'Administration et plusieurs autres, 
constituant une sorte d'administration parallèle dépendant directement du président.

Face à ce type d'administration, on retrouve les chefs de département de la bureaucratie permanente, dont notamment le Département d'État, machine particulièrement complexe, lourde et lente dans ses opérations, comportant toute une série de bureaux spécialisés auxquels on reproche leur incapacité à garder secrètes les opérations les plus délicates. D'où la tendance à vouloir les contourner tant au niveau de l'élaboration que de l'exécution d'un certain nombre de politiques extérieures, en ayant recours à une institution telle que le C.N.S.

Dans le cas de tous les organismes précités, il s'agit d'un modèle oligarchique (oligarchical model) ${ }^{8}$ en contact avec des groupes d'intérêt et des institutions privées situés en dehors du système politique.

Mais, cette oligarchie qui occupe les postes clef dans les organes précités, tout en partageant un certain consensus sur les objectifs de base du président, apparaît souvent divisée quand aux moyens et méthodes à utiliser. Phénomène que vient confirmer l'Irangate où l'on voit s'affronter divers conseillers d'autant plus divisés entre eux que personne ne tient à revendiquer la paternité d'un échec d'une telle envergure. Ceci semble d'ailleurs confirmer une autre constante: il y a une corrélation directe entre les apparences d'unité décisionnelle et le succès des politiques. En d'autres termes, les cas d'échecs graves servent en quelque sorte de révélateur des divisions internes des conseillers et des organes de conseil. Et souvent, dans ce cas, le Président aura tendance à chercher un bouc émissaire limité à une institution et quelques individus isolés artificiellement.

8. Thomas Dye, Who's Running America? Institutional Leadership in the United States, New Jersey, Prentice Hall, 1976, voir "An Oligarchical Model of National Policy Making», p. 196-197. 


\section{Variables et constantes du mode de gestion présidentielle}

Finalement, qui est responsable dans un tel système? Le Président évidemment puisqu'il est le seul élu. Et pourtant, comme on l'a rappelé plus haut, il existe une foule de problèmes cruciaux où il est absolument forcé de déléguer en pratique beaucoup de pouvoirs et où, dans la très grande majorité des cas, il est incapable de contrôler ce qui se fait, sauf dans quelques dossiers majeurs qu'il peut se réserver. D'où une question fondamentale à poser ici: dans ces conditions, l'art de gouverner n'est-ce pas avant tout être capable de choisir les problèmes clefs de la politique intérieure et extérieure. Ce qui implique beaucoup de jugement politique et en même temps un pouvoir de concentration exceptionnel afin de pouvoir en analyser toutes les ramifications.

Étant donné le caractère à la fois politique et technique de tels dossiers, le Président se doit de consulter les conseillers les plus compétents de son entourage. Mais, dans une affaire comme celle ressortant de l'Irangate, de quels conseillers et de quels organes de conseil s'agit-il?

Et ici, on effectue un autre constat, relatif cette fois à la variable personnalité 9 : les présidents ont un style et une façon personnelle d'utiliser leurs organes de conseil. Si on se limite aux présidents après la seconde guerre mondiale, ceux classifiables parmi les moins actifs semblent être Eisenhower et Reagan en

9. James Barber a établi 4 catégories de présidents: actif positif, actif négatif, passif positif et passif négatif, voir The Presidential Character, New Jersey, Prentice Hall, 1972. Erwin Hargrove se contente de deux grandes catégories: président of action et président of restraint. Kennedy, par exemple, se situe dans la première et Eisenhower dans la seconde. Voir: Présidential Leadership, Personality and Political Style, New-York, Macmillan, 1968. 
raison de leur implication moindre dans les dossiers (comparativement aux autres présidents) et leur tendance à déléguer proportionnellement plus de pouvoirs à certains conseillers ${ }^{10}$, voire à choisir un ou deux de ceux-ci comme "chef(s) d'état-major». Eisenhower, par exemple, s'est essentiellement appuyé sur J. F. Dulles (secrétaire du Département d'État) pour les affaires étrangères (surtout lors du premier terme) tandis que Sherman Adams (du Bureau de la Maison blanche) était son conseiller responsable de la politique intérieure et ce, souvent jusque dans les moindres détails.

Le Président Reagan a hésité plus longtemps à cet égard. En 1982, lorsque Haig (qui aurait voulu jouer le rôle de F. Dulles et de Kissinger) démissionne, on ne sait pas très bien qui dirige la politique extérieure, on parle de troika californienne, les conseillers spéciaux pour le Conseil national de Sécurité se succèdent après des affrontements avec d'autres départements (défense, affaires étrangères) et il règne une certaine confusion. Finalement cependant James Baker (membre de la troika précitée) émerge comme «chief of staff» (durant le premier mandat notamment) suivi de Donald Regan dont les carences dans cette fonction difficile l'obligeront à démissionner au profit d'un autre chef d'état-major: Howald Baker (du Bureau de la Maison blanche également).

Contrairement aux "présidents d'action» (au sens conféré par Hargrove) les deux présidents républicains précités ont donc tendance à favoriser un modèle de gestion plus hiérarchisé en laissant à ce ou ces conseillers clefs le soin de coordonner les politiques. Mais à partir de quelle base institutionnelle pour la

10. Ce qui pour Stanley Hoffman ne signifie pas nécessairement que le Président ne contrôle pas ses conseillers. Au contraire, selon lui, après les oscillations de plusieurs de ses joueurs, il "reprend les rênes le moment venu, après avoir tenu compte de ce qui reste sans doute l'essentiel: l'opinion intérieure, etc». Mais ceci a été écrit un peu avant l'Irangate. Voir, "Les États-Unis», Pouvoirs, (Revue française d'études constitutionnelles et politiques), 1984 no 29 , p. 57. 
politique étrangère? Le Département d'État (sous Truman et Eisenhower) ou le Conseil national de Sécurité?

À première vue, cette dernière institution (créée en 1947) a pour principaux objectifs justement de formuler et coordonner l'application d'une politique d'ensemble en matière de sécurité nationale, impliquant donc la défense, la politique extérieure et de nombreux aspects (directs ou indirects) de la politique intérieure reliée à ces deux volets inséparables. Le C.N.S. regroupe d'ailleurs notamment les titulaires des départements de la défense et des affaires étrangères et il comporte de nombreux groupes et commissions de travail spécialisés. Ces organes collectent, trient, coordonnent les données et font des recommandations au responsable du C.N.S. Quant aux chefs des départements, ils disposent d'énormes ressources en matière de renseignements et de moyens d'exécution mais ils ont tendance à donner piorité aux objectifs (et aux méthodes) de leur propre organisation et s'efforcent d'étendre leurs pouvoirs et influence. À cela s'ajoutent les lacunes soulignées plus haut quant au mode de fonctionnement d'un organisme tel que le Département d'État.

Dans ces conditions, ici aussi le Président aura tendance à réduire le nombre des conseillers participant aux réunions les plus importantes pour l'élaboration des politiques. Comme le Président Truman il sera tenté de constituer une sorte d'État-major (Senior Staff) devant assurer une meilleure liaison avec les départements et agences gouvernementales ou encore, et ceci comporte plus de risques, il sera porté à se fier à un seul responsable. Ce qui s'avère plus simple, à condition de disposer d'un conseiller d'une valeur exceptionnelle. Sans nécessairement reconnaître une telle qualité à Kissinger, on connaît en tout cas le rôle capital qu'il a joué lorsqu'il était responsable du Conseil national de Sécurité, avant de passer à la direction du Département d'État où il a pu appliquer 
les politiques initiées au dit C.N.S. lors du premier mandat présidentiel.

En matière de défense et de politique extérieure (intrinsèquement liées), le C.N.S., constitue-t-il actuellement le mécanisme décisionnel par excellence ${ }^{11}$ ? On a souvent écrit que ce dernier, grâce à sa souplesse et à la légèreté de ses structures, permet des mouvements rapides et secrets, tant dans l'initiative de certaines politiques que dans la coordination de leur application.

En réalité, l'utilisation de ce mécanisme varie selon les présidents en place. Kennedy, contrairement à Eisenhower, l'utilise peu, il recourt davantage à de nombreuses "task forces", équipes mobiles, composées de personnes ressources diversifiées, s'appuyant sur un état-major d'une dizaine de personnes de grand talent. Parmi celles-ci émergent des conseillers tels que Bundy, Sorensen, Mac Namara, etc., dans un cadre peu hiérarchisé. Il s'agit manifestement d'un modèle de gestion très différent, au sein duquel le C.N.S. ne constitue plus le centre de décision principal.

Par contre, sous Nixon, le C.N.S. est revitalisé, le Président s'appuie à la fois sur son état-major des affaires intérieures (domestic Affairs Staff) et le dit C.N.S., étroitement contrôlé par Kissinger et où l'on a reconstitué des groupes, commissions et comités permanents ou ad hoc effectuant un travail de préparation sans comparaison possible avec ce qui se passe sous Eisenhower. Symboliquement, à cette époque le Conseiller responsable du C.N.S. (Kissinger) surclasse et court circuite le responsable du Département d'État (Rogers).

Le modèle républicain (hiérarchisé) d'Eisenhower et de Nixon est-il repris par leur successeur Reagan? L'affaire Irangate et la succession de cinq assistants responsables du C.N.S., sous sa

11. Dans une étude remarquable, Destler avait, il y a déjà longtemps, souligné le danger d'affaiblir le Département d'État au profit du C.N.S. Voir Presidents Bureaucrats and Foreign Policy, Princeton University Press, 1972. 
présidence, semblent confirmer un manque de contrôle ${ }^{12}$ sur le processus décisionnel alors qu'il avait à sa disposition un secrétaire d'État (Shultz) disposant d'une expérience exceptionnelle tant en matière de politique intérieure qu'en matière de politique extérieure.

\section{Carences de la coordination et style Reagan}

À la suite de ce survol, nous pourrions tirer quelques conclusions permettant de faire le point avant de nous concentrer davantage sur la variable personnalité, un peu mieux délimitée cette fois.

Tout d'abord, on l'a vu, l'utilisation du C.N.S., varie considérablement d'un président à l'autre. L'on est tenté de dire que les présidents moins actifs (dans le sens repris plus haut) ont tendance à s'en servir plus systématiquement mais une telle conclusion simplifierait grandement les choses. Ainsi, Nixon, Président particulièrement dynamique en politique étrangère, l'a fortement utilisé lors de son premier mandat (au cours duquel il procède à des manœuvres relativement audacieuses, en direction de la Chine notamment). Ensuite, il s'appuiera davantage sur le Département d'État pour appliquer les politiques amorcées dans le cadre précité.

En second lieu, plusieurs observateurs pensent que le choix d'un ou de deux chefs d'état major (l'un pour les affaires intérieures, l'autre pour les affaires extérieures) serait la caractéristique des présidents moins actifs (voire passifs). Alors que les plus dynamiques s'entourent davantage d'une équipe, voire de différentes équipes à première vue plus difficiles à contrôler mais plus créatives. Ceci aussi mérite d'être nuancé mais, dans tous les cas, désormais les principaux conseillers sont davantage intégrés dans l'organisation

12. C'est une opinion que, pour notre part, nous avions déjà exprimée, lors de la «démission» du général Haig, dans un article intitulé: «Le cas Haig, un épiphénomène inquiétant pour l'avenir». Le Devoir, 9 juillet 1982, page l'actualité. 
officielle de la présidence et ils proviennent majoritairement des grandes entreprises privées.

Autre constat: la nécessité d'un instrument permanent de coordination au niveau de l'élaboration et du contrôle de l'exécution des politiques se fait sentir plus que jamais avec la complexité croissante des problèmes internes et externes. Or, cet instrument, malgré toutes les tentatives, en théorie et sur le terrain, n'a pas encore été mis au point. Il devrait répondre plus adéquatement aux exigences d'une programmation à long terme (dépassant les préoccupations électorales), tout en restant souple et mobile, en comparaison avec l'appareil bureaucratique permanent (départements, etc.) qui a d'autres fonctions et une autre vision des problèmes. Un tel instrument devrait être suffisamment puissant et influent pour intégrer dans ses rouages les principaux responsables de l'«autre administration".

Quelle que soit la personnalité du Président au pouvoir, il se heurte aux problèmes de fonds rappelés plus haut. Il y répondra avec un succès variable et au milieu de difficultés croissantes.

Le Président Reagan confirme ce dernier constat, en dépit du fait que la conjoncture économique et politique lui ayait été très favorable à la fin du premier mandat et au début du second. À première vue, il disposait d'atouts majeurs et semblait devoir terminer son mandat avec succès. C'est du moins l'image qui était projetée dans l'opinion publique américaine.

En réalité, comme on le signalait plus haut, la fonction est devenue de plus en plus écrasante («a killing job») ${ }^{13}$. Et ce, à un moment où les États-Unis connaissent un déclin relatif et sont confrontés avec des problèmes internes cruciaux que la vision optimiste du Président ne peut pas toujours masquer (l'on pense

13. À noter que depuis F. Roosevelt, à part Eisenhower, aucun président n'a rempli deux mandats au complet. Et le second mandat de Reagan est sérieusement hypothéqué. 
en particulier au problème de la pauvreté dans les grandes villes et aux disparités économiques régionales).

Or, face à cette situation, nous avons actuellement un président qui, et c'était évident dès le départ, n'était ni préparé ni équipé pour y faire face. D'une certaine façon pourtant, on ne peut lui reprocher un manque total de vision ${ }^{14}$, même si celle-ci s'exprimait en quelques thèmes et slogans simplifiés répondant aux besoins d'une partie de l'opinion publique.

Une des clefs de ses succès initiaux réside dans le fait qu'il a, pendant un certain temps, laissé l'impression que malgré quelques incidents de parcours il savait ce qu'il voulait: restaurer le prestige des États-Unis (affaiblis, selon lui, sous Carter), effectuer une reprise économique (dans une perspective proche d'un Milton Friedman hypersimplifié), rétablir la suprématie militaire américaine, le tout dans un dénominateur commun aggressif: l'anticommunisme.

Ceci impliquait deux mouvements à première vue corrélatifs: un ralentissement des interventions de l'État dans l'économie et le social (ce que le budget ne put cependant traduire) et un renforcement de l'État militaire. Sur ce dernier plan, la politique de défense devait automatiquement s'accompagner d'une politique étrangère plus dure, notamment à l'égard de petits pays comme la Libye et le Nicaragua (relié également à l'Irangate). Ces derniers étant perçus et présentés comme constituant une menace pour le système de défense des États-Unis.

Rendu à ce stade de l'exposé, une autre question, (d'ordre comparatif cette fois), se pose ici. L'Irangate n'est-il pas que la pointe de l'iceberg de politiques pratiquées aux Étas-Unis et dans

14. Contrairement à ce que l'on affirme trop vite dans certains manuels sur les États-Unis, la plate-forme électorale d'un parti peut être précise. Et la force de R. Reagan c'est d'avoir appliqué l'esprit et une partie importante du programme de la Convention républicaine de 1980 . Pour une synthèse de celles-ci, voir: «Extraits de la plate-forme républicaine 1980 ", Information USA Document, Washington, D.C., 3/10/1980, 10 p. 
bien d'autres pays, au cours desquelles l'on effectue un nombre considérable d'opérations illégales ou en marge de la légalité? En fait, l'affaire Irangate est peu de chose à côté de la multitude d'opérations de la nature précitée, effectuées avant, pendant et après celle-ci. Les esprits un peu cyniques diront alors que le seul crime c'est de se faire prendre.

Si l'on poursuit un tel raisonnement jusqu'au bout, l'on dira alors que le Président a été malchanceux ou maladroit en recourant à des moyens inefficaces pour atteindre une ou des fins conformes à sa politique. Quoiqu'il en soit, il est probable qu'en cas de réussite des politiques pratiquées (tant à l'égard de l'Iran que du Nicaragua), la majorité des critiques se serait montrée moins préoccupée du choix des moyens utilisés. Car si, suivant l'adage machiavélien, la fin justifie les moyens, l'on peut dire aussi que trop souvent le succès et l'efficacité justifient tout, spécialement dans les rapports entre États. Et davantage encore lorsque les dits rapports sont perçus d'une façon aussi manichéenne que ne le projettent l'image et le discours du Président actuel.

Une autre hypothèse serait qu'il ait choisi des conseillers incompétents et qu'ils aient échappé à son contrôle. Dans ce cas c'est la personnalité et le style du Président qui est encore en question et non le bien-fondé des politiques. Et c'est sur ces éléments que l'attention risque le plus de s'attarder aux dépens des problèmes de fond rappelés plus haut. De fait, dans la presse américaine, depuis l'Irangate, on retrouve un nombre considérable d'articles faisant allusion aux carences physiques et intellectuelles du Président. À un certain moment, le processus faisait boule de neige et risquait d'atteindre un point de non retour si un certain consensus de retenue ne s'établissait pas, à la fois au Congrès et dans les media d'information. À ce jour, la somme de critiques 
formulées ${ }^{15}$ est particulièrement lourde et son accumulation s'est faite en un temps record.

Et pourtant, il y a déjà longtemps que certains observateurs de la scène politique américaine, tout en reconnaissant les dons de communicateur exceptionnel du Président Reagan, ont souligné son manque de connaissance, voire d'intérêt, pour les dossiers les plus importants, spécialement ceux des affaires extérieures. On a souvent prétendu qu'en se limitant aux grandes lignes de la politique, il pouvait mieux la contrôler. Ceci implique qu'il doit déléguer beaucoup de pouvoirs en pratique à ses conseillers ${ }^{16}$ et ne pas s'immiscer dans les détails. Mais d'autre part, l'on souligne aussi à quel point il perd un temps précieux à s'attarder à des détails ou des dossiers mineurs sans rapport avec une fonction aussi lourde et à laquelle, finalement, il consacre peu d'énergie, surtout si on le compare avec les présidents qui l'ont précédé depuis Coolidge.

En fin de compte, l'échec de l'Irangate, se situe à plusieurs niveaux: celui des institutions devant conseiller le Président, celui de la personnalité de certains acteurs publics (conseillers incompétents) et enfin celui du Président seul responsable en dernier ressort. C'est peut-être aussi un échec plus grave, résultant d'un système politique éprouvant beaucoup de difficulté à sélectionner les dirigeants les plus valables alors que le pays dispose certainement du potentiel nécessaire ${ }^{17}$. À cet égard l'Irangate aura alors joué

15. Exemples parmi d'autres en 1986: Max Holland, Tom Wicker et Reston (au New York Times) et même dans le Time Magazine, Hugh Sidey. Voir plus particulièrement l'article de Richard Stengel, "How Reagan stays out of touch", (Time, 8/ $12 / 1986$, p. 32), accréditant l'idée d'une incompétence manifeste sauf au niveau du marketing d'idées, conçues par d'autres que R. Reagan, à part quelques grandes orientations générales.

16. Parmi les études consacrées à cette question, voir, entres autres notre livre: La Présidence moderne aux États-Unis, Personnalité et institutionnalisation, Presses de l'Université du Québec, 1974.

17. Pour une interprétation américaine (à la foi originale et réaliste) du dit système, voir notamment: Edward Greenberg, The American Political System. A Radical Approach, Boston, Little Brown and Company, 1983, 457 p. 
un rôle de révélateur qui ne manque pas d'être inquiétant pour tous ceux qui de près ou de loin dépendent du fonctionnement du dit système, pour le meilleur ou pour le pire.

Cet article visait donc à reprendre quelques facteurs d'ordre personnel et d'ordre systémique devant permettre de mieux situer (et non d'expliquer) l'affaire «Irangate».

Dans une telle perspective, l'on tend à considérer que le scandale précité semble découler assez logiquement de certaines lacunes relevant plus du fonctionnement du système politique que des individus qui en sont à la fois les acteurs et le produit. C'est là un point de vue certes discutable et qui mériterait évidemment une analyse beaucoup plus poussée pour que la démonstration soit valable sinon claire.

Quant aux réformes à apporter, elles dépendent aussi de l'analyse que l'on fait de ce système politique et en particulier de son institution présidentielle. À titre documentaire, signalons un article d'un spécialiste de ce genre de question: James Mac Gregor Burns ${ }^{18}$. Suite à l'Irangate, il préconise une série de transformation profondes des institutions politiques des États-Unis. Ces réformes ${ }^{19}$ semblent s'inspirer de plusieurs principes de base d'un régime parlementaire idéal. Mais, c'est là un autre problème soulevé à l'occasion d'un scandale qui, à plusieurs égards, n'est guère surprenant tout compte fait.

18. "A Call for Reform", Life-Time, 1987, Fall, Special Issue: The Constitution, p. 126.

19. Renforcer l'impeachment, le prévoir pour les cas de nette incapacité de diriger le pays, surtout quand le président a perdu la confiance du pays, exemples: Watergate, Irangate. Permettre de nommer dans l'administration des membres du Congrès pour y apporter le point de vue de la base. Renforcer systématiquement le rôle du parti d'opposition. Réduire les possibilités des «accords exécutifs» qui limitent les pouvoirs de contrôle du Congrès, etc. 